\title{
Small-Scale Spectrum of a Scalar Field in Water: The Batchelor and Kraichnan Models
}

\author{
Xavier Sanchez, Elena Roget, Jesus Planella, and Francesc Forcat \\ Department of Physics, University of Girona, Girona, Spain
}

(Manuscript received 27 January 2011, in final form 9 June 2011)

\begin{abstract}
The theoretical models of Batchelor and Kraichnan, which account for the smallest scales of a scalar field passively advected by a turbulent fluid (Prandtl $>1$ ), have been validated using shear and temperature profiles measured with a microstructure profiler in a lake. The value of the rate of dissipation of turbulent kinetic energy $\varepsilon$ has been computed by fitting the shear spectra to the Panchev and Kesich theoretical model and the one-dimensional spectra of the temperature gradient, once $\varepsilon$ is known, to the Batchelor and Kraichnan models and from it determining the value of the turbulent parameter $q$. The goodness of the fit between the spectra corresponding to these models and the measured data shows a very clear dependence on the degree of isotropy, which is estimated by the Cox number. The Kraichnan model adjusts better to the measured data than the Batchelor model, and the values of the turbulent parameter that better fit the experimental data are $q_{B}=$ $4.4 \pm 0.8$ and $q_{K}=7.9 \pm 2.5$ for Batchelor and Kraichnan, respectively, when Cox $\geq 50$. Once the turbulent parameter is fixed, a comparison of the value of $\varepsilon$ determined from fitting the thermal gradient spectra to the value obtained after fitting the shear spectra shows that the Kraichnan model gives a very good estimate of the dissipation, which the Batchelor model underestimates.
\end{abstract}

\section{Introduction}

Since Osborn (1980), turbulent fluxes in sheared stratified flows have been determined from the turbulent kinetic energy dissipation rate $\varepsilon$; the stratification $N^{2}$; and the so-called mixing efficiency $\Gamma$, which for developed turbulence can be considered a constant value (Dillon 1982; Oakey 1982, Smyth et al. 2001). Accordingly, to accurately determine the turbulence fluxes in natural aquatic systems (Lozovatsky et al. 1999), $\varepsilon$ also has to be obtained precisely. The value of $\varepsilon$ can be obtained from small-scale shear measurements (Gargett et al. 1984; Gregg and Horne 2009), indirectly from microtemperature data (Jonas et al. 2003; Sanchez and Roget 2007), or from both methods (Kocsis et al. 1999). Field measurements of microstructure temperature and smallscale shear in a natural aquatic system are usually recorded by fast-response thermistors and airfoils mounted on profilers or other types of underwater vehicles. Some of these profilers, like the Self-Contained Autonomous Microprofiler (SCAMP; Stevens et al. 1999), measure only

Corresponding author address: Xavier Sanchez, Department of Physics, University of Girona, Campus Montilivi PII, Girona, Catalonia 17071, Spain.

E-mail: xavier.sanchez@udg.edu microtemperature data, but others, like the MicrostructureTemperature profiler (MST; Prandke and Stips 1998) used here, measure microtemperature and small-scale shear.

Once statistically homogeneous turbulent segments are determined within each measured profile (Piera et al. 2002), the one-dimensional (1D) spectrum of the measured small-scale shear or the microtemperature gradient can be calculated and fitted to the one-dimensional spectrum model for isotropic turbulence, assuming local isotropy within the homogeneous segments (Roget et al. 2006). Because temperature and shear spectrum models depend on $\varepsilon$, it can be obtained from the best fit between the experimental and the theoretical spectra. The temperature spectrum model also depends on the rate of dissipation of temperature variance $\varepsilon_{\theta}$, but this can be obtained by integrating the temperature gradient spectrum (Luketina and Imberger 2001).

The theoretical energy spectrum model of Panchev and Kesich (1969, hereafter PK69) describes the turbulent velocity within the isotropic universal equilibrium range, covering the inertial and viscous subranges (Tennekes and Lumley 1972). The PK69 model has been widely accepted within the oceanographic community (Gregg 1999), which also uses the very similar Nasmyth (1970, hereafter N70) model obtained experimentally. Regarding 
microstructure temperature, the spectral model proposed by Batchelor (1959, hereafter B59) is widely accepted and extensively used in oceanic and limnic waters to obtain $\varepsilon$ (Wüest et al. 1996; Saggio and Imberger 2001), but lately proposals have been made (Nash and Moum 2002; Roget et al. 2006; Sanchez and Roget 2007) to consider the alternative model given by Kraichnan (1968, hereafter K68).

The main purpose of this study is to check which of the models, B59 or K68, more closely follows the microstructure thermal data recorded in a lake. Both models stand for the three-dimensional spectra $E_{\theta}(k)$ of a conserved scalar field that is passively advected by an incompressible turbulent fluid with a Prandtl number $\operatorname{Pr}=\nu / \kappa_{\theta}$ greater than 1 , where $k$ is the circular wavenumber (in $\mathrm{rad} \mathrm{m}^{-1}$ ) and $v$ and $k_{\theta}$ are the viscosity and the diffusivity of the scalar, respectively. Also, both of them assume homogeneous, isotropic, and stationary turbulence and describe the spectra for those wavenumbers above the inertial-convective subrange (Obukhov 1949; Corrsin 1951), where $E_{\theta} \propto k^{-5 / 3}$, as is also the case of the energy spectra in the inertial subrange, where $E \propto k^{-5 / 3}$ (Kolmogorov 1941).

The scalar spectra $E_{\theta}$ in both theoretical models (B59 and K68) can be scaled by the so-called Batchelor wavenumber $k_{d \theta}=\left[\varepsilon /\left(\nu \kappa_{\theta}^{2}\right)\right]^{1 / 4}$, which is related to the Kolmogorov wavenumber $k_{d}=\left(\varepsilon / \nu^{3}\right)^{1 / 4}$ and the Prandtl number as in the equation $k_{d \theta}=k_{d} \operatorname{Pr}^{1 / 2}$. More precisely, the scalar spectrum depends on $E_{\theta}\left(k, \phi, \varepsilon_{\theta}, k_{\theta}\right)$, where $\phi=k_{d \theta} / \sqrt{2 q}$ and $q$ is the so-called turbulent parameter, usually considered as a constant of the model. Hereafter, we will use $q_{B}$ and $q_{k}$ when referring to the turbulent parameter in the B59 and K68 models, respectively. Although the B59 and K68 models consider different physical hypotheses, both models contain the power law $E_{\theta} \propto k^{-1}$ for the smallest wavenumbers (the viscousconvective subrange). Near $k_{d \theta}$, which is larger than $k_{d}$ for $\operatorname{Pr}>1$, the scalar spectrum presents a cutoff, corresponding to the transition into the viscous-diffusive subrange. The exact value of this cutoff wavenumber depends on the model and on the value of $q$.

Given the $3 \mathrm{D}$ spectrum model $E_{\theta}$, the corresponding one-dimensional spectrum of the scalar gradient, which is the spectrum that is usually fitted to recorded field data, presents a dependence similar to $E_{\theta}$, which is $F_{\partial \theta}\left(k_{3}, \phi, \varepsilon_{\theta}, \kappa_{\theta}\right)$, where now $k_{3}$ is one of the Cartesian components of the wavenumber (e.g., the third). One of the aims of this paper is to check the values of the turbulent parameters that would give a better fit of our experimental data to the B59 and K68 models. This will be possible by considering the value of the turbulent kinetic energy dissipation rate $\varepsilon$ computed from simultaneously measured small-scale shear data.
The validity of the velocity and scalar theoretical spectral models discussed in this paper depends on the degree of isotropy. The degree of isotropy of the turbulent flux can be evaluated from the buoyancy Reynolds number $\operatorname{Re}_{b}=\varepsilon /\left(\nu N^{2}\right)$, where $N$ is the Brunt-Väisälä frequency, or from the Cox number $C_{x}=\varepsilon_{\theta} /\left(2 \kappa_{\theta} \bar{T}_{z}^{2}\right)$, with $\bar{T}_{z}$ being the mean vertical stratification of the scalar field within the considered segment. Dillon and Caldwell (1980) argued that $\operatorname{Re}_{b} \propto \operatorname{Pr}^{-1} C_{x}$ and observed that the viscous-convective power-law subrange of the scalar spectra $E_{\theta} \propto k^{-1}$ appears only with large values of $C_{x}$. Considering these two nondimensional numbers, we have evaluated the validity of the B59 and K68 models for the temperature gradient spectrum, depending on isotropic conditions.

In this paper, the theoretical basis of the B59 and K68 models and the references where they have been validated are reviewed in section 2 . In section 3 , we present the measurements we performed for this study and the procedure used to process the data: (i) determine turbulent homogeneous segments where the small-scale shear spectrum follow the universal form of the PK69 model and obtain the rate of dissipation of turbulent kinetic energy $\varepsilon$; (ii) determine the rate of dissipation of the scalar variance $\varepsilon_{\theta}$ by integrating the one-dimensional spectrum of the temperature gradient; and, once $\varepsilon$ and $\varepsilon_{\theta}$ have been calculated for every segment, (iii) the resulting thermal gradient spectra are fitted to the B59 and K68 models varying the value of the turbulent parameter $q$. In section 4, the results are presented and the $q$ value statistics obtained are discussed. Alternatively, the bin-averaged nondimensional spectrum for the ensemble of segments is computed and compared to the nondimensional B59 and K68 models to also obtain the turbulent parameter for each model. Differences between the values of $\varepsilon$ computed either from the shear spectra or from the scalar spectra are discussed. Finally, we discuss which model follows the experimental data better, the importance of the degrees of isotropy for a good fit of the experimental data to the theoretical models, and the validity of a constant value for the mixing efficiency of isotropic turbulence.

\section{The universal scalar spectrum}

\section{a. Theoretical background}

The physical hypotheses of the B59 model for the 3D scalar spectrum $E_{\theta}(k)$ are mainly about the space and time variability of the turbulent strain rate tensor (Kundu 2008). These are as follows: (i) the strain rate is locally uniform (without space variability) for scales smaller than the Kolmogorov length; (ii) within these small scales the principal rates of strain $(\alpha, \beta$, and $\gamma)$ and their 
corresponding directions evolve slowly over time in comparison with a time scale of $1 /|\gamma|$, where $\gamma$ is the least principal rate of strain, which is negative because $\alpha+\beta+\gamma=0$ (for incompressible fluids); and (iii) the value of $\gamma$ can be statistically assumed as an effective value $\gamma_{\text {eff }}$ without fluctuations. If the strain rate is uniform and evolves slowly, most of the time the gradients of the scalar field will be locally aligned with the direction of the least principal rate of strain due to the advective effect of the flux on the scalar field.

Under statistically stationary turbulence, the time evolution of the temperature spectrum (Hinze 1975) is given by

$$
\partial E_{\theta}(k) / \partial t=T_{\theta}(k)-D_{\theta}(k)=0
$$

where $D_{\theta}=2 \kappa_{\theta} k^{2} E_{\theta}$ is the dissipation spectrum of the scalar field and $T_{\theta}(k, t)$ is the transfer spectrum, which stands for the nonlinear variance flux of the wavenumber $k$ from the rest of the wavenumbers. Following Batchelor's hypothesis, the transfer spectrum $T_{\theta}(k, t)$ can be related with the three-dimensional scalar spectrum $E_{\theta}(k, t)$ as

$$
T_{\theta}=\gamma_{\text {eff }} \partial\left(k E_{\theta}\right) / \partial k,
$$

and Eq. (1) can be solved to explicitly determine $E_{\theta}(k)$. At small scales, the variance of the partial derivatives of the velocity is proportional to $(\varepsilon / \nu)$, which can be checked dimensionally, and the effective least principal rate of strain $\gamma_{\mathrm{eff}}$, according to Batchelor, is proportional to $(\varepsilon / \nu)^{1 / 2}$ so that

$$
\gamma_{\mathrm{eff}}=-q_{B}^{-1}(\varepsilon / \nu)^{1 / 2},
$$

where $q_{B}$ is the nondimensional turbulent parameter in the B59 model.

K68 and Kraichnan (1974) proposed an alternative to Batchelor's model. Kraichnan considered rapid fluctuations in time of the velocity field that have an infinitely short correlation time compared to the convective and diffusive time scales. Under this hypothesis, the transfer spectrum is

$$
T_{\theta}=-A(t) / 5 \partial\left[k E_{\theta}-k / 3 \partial\left(k E_{\theta}\right) / \partial k\right] / \partial k,
$$

where $A(t)=\int_{-\infty}^{t}\left\langle\partial u_{i}(\mathbf{x}, t) / \partial x_{n} \partial u_{i}\left(\mathbf{x}, t^{\prime}\right) / \partial x_{n}\right\rangle d t^{\prime}$ and $u_{i}(\mathbf{x}, t)$ is the turbulent velocity field at space position $\mathbf{x}$ and time $t$; repeated indexes mean summation and \langle\rangle is an ensemble average. When statistical stationarity and homogeneity are assumed, $A$ is a constant that K68, like Batchelor, scaled as $A=5 q_{K}^{-1}(\varepsilon / \nu)^{1 / 2}$, where $q_{K}$ is the turbulent constant for the spectrum model.
Using Eqs. (2) and (4) to integrate Eq. (1) gives the forms of the Batchelor and Kraichnan spectra of the scalar. Both spectrum models can be nondimensionalized with the following scaling, called Batchelor scaling:

$$
E_{\theta}\left(k, \phi, \varepsilon_{\theta}, \kappa_{\theta}\right)=\frac{\varepsilon_{\theta}}{2 \kappa_{\theta} \phi^{3}} \hat{E}_{\theta}(y),
$$

where the nondimensional spectrum $\hat{E}_{\theta}(y)$ depends on the nondimensional wavenumber $y=k / \phi$ with $\phi=k_{d \theta} / \sqrt{2 q}$. In the case of the Batchelor and Kraichnan models, the nondimensional spectrum $\hat{E}_{\theta}(y)$ takes the forms (superscripts $B$ and $K$, respectively)

$$
\begin{aligned}
& \hat{E}_{\theta}^{B}(y)=y^{-1} \exp \left(-y^{2} / 2\right) \text { and } \\
& \hat{E}_{\theta}^{K}(y)=\frac{1+\sqrt{3} y}{y} \exp (-\sqrt{3} y),
\end{aligned}
$$

and their shape depends on the respective turbulent parameters $q_{B}$ and $q_{K}$.

The cutoff in the viscous-diffusive subrange for both models is better analyzed from the dissipative spectrum $D_{\theta}=2 \kappa_{\theta} k^{2} E_{\theta}$, because it presents a very clear maximum at the cutoff. Figure 1 shows the dissipative spectra for the B59 and K68 models in the nondimensional form $D_{\theta} k_{d \theta} / \varepsilon_{\theta}$ as a function of two nondimensional wavenumbers, $\hat{k}=k / k_{d \theta}$ (lower horizontal axis) and $\tilde{k}=k / k_{d}$ (upper horizontal axis), where $k_{d}$ and $k_{d \theta}$ are the Batchelor and the Kolmogorov wavenumbers introduced in section 1 . As observed, for the same $q$ both spectra coincide for the lower wavenumbers. However, the dissipation maximum, which is one order of magnitude less than $k_{d}$, varies from one model to the other and for different $q$ values. The maximum in Fig. 1 for the value $q=4$ is $\hat{k}=0.36$ for B59 (solid line) and $\hat{k}=0.33$ for $\mathrm{K} 68$ (dashed line); for $q=8$, it is at $\hat{k}=0.25$ for B59 (dotted line) and $\hat{k}=0.23$ for K68 (dashed-dotted line). In general, the $\hat{k}$ value where the dissipation spectrum has its maximum is $0.7109 / \sqrt{q}$ for B59 and $0.6573 / \sqrt{q}$ for K68.

As was discussed in section 1, the measurable spectrum is usually the one-dimensional gradient spectrum $F_{\partial \theta}\left(k_{3}\right)$, which depends on the component of the wavenumber vector in one direction: for example, the third coordinate $k_{3}$. A consequence of isotropy is that the onedimensional spectra formed by the other two Cartesian directions have a shape identical to $F_{\partial \theta}\left(k_{3}\right)$. The spectrum $F_{\partial \theta}\left(k_{3}\right)$ is obtained from $E_{\theta}(k)$ (Hinze 1975; Gibson and Schwarz 1963) and can be nondimensionalized based on the following scaling:

$$
F_{\partial \theta}\left(k_{3}\right)=\frac{\varepsilon_{\theta}}{2 \kappa_{\theta}} \frac{1}{\phi} f_{\partial \theta}\left(y_{3}\right)=\frac{\varepsilon_{\theta}}{2 \kappa_{\theta} k_{d \theta}} \sqrt{2 q} f_{\partial \theta}\left(y_{3}\right),
$$






FIG. 1. Dissipation spectra of a passive scalar in nondimensional form $D_{\theta} k_{d \theta} \varepsilon_{\theta}{ }^{-1}$ as a function of the Batchelor nondimensional wavenumber $\hat{k}=k \times k_{d \theta}^{-1}$ (lower horizontal axis) and the Kolmogorov nondimensional wavenumber $\tilde{k}=k \times k_{d}^{-1}$ (upper horizontal axis). The curves show the B59 and K68 models for two values of the turbulent parameter, $q=4$ and $q=8$.

where $y_{3}=k_{3} / \phi$ with $\phi=k_{d \theta} / \sqrt{2 q}$ and $f_{\partial \theta}\left(y_{3}\right)$ is the nondimensional function, which, for the Batchelor and Kraichnan models, are

$$
f_{\partial \theta}^{B}\left(y_{3}\right)=y_{3}\left\{\exp \left(-y_{3}^{2} / 2\right)-y_{3} \sqrt{\frac{\pi}{2}}\left[1-\operatorname{erf}\left(y_{3} / \sqrt{2}\right)\right]\right\}
$$

and

$$
f_{\partial \theta}^{K}\left(y_{3}\right)=y_{3} \exp \left(-\sqrt{3} y_{3}\right)
$$

respectively.

\section{b. Experimental background}

The first experimental value for the B59 turbulent parameter was found to be $q_{B} \approx 2$ and was given in the same article where the model was introduced (B59) based on previous laboratory results for the estimate of the average value of the least principal rate of strain. Gibson (1968), hypothesizing that the effective value $\gamma_{\text {eff }}$ is bounded by the extreme values of the volume averaging of the local least principal rate of strain $\gamma$, predicted a theoretical range of $\sqrt{3}<q_{B}<2 \sqrt{3}\left(1.73<q_{B}<3.46\right)$.

The first estimate of $q_{B}$ based on field data was given by Grant et al. (1968) using 12 turbulent patches recorded in the open sea and in a tidal channel. As the authors pointed out, the determination of $q$ is very sensitive to instrumental errors and to nonlocal isotropy, so before giving $q$ a value they disregarded 3 out of 12 spectra to ensure that the corresponding turbulent velocity followed a universal isotropic spectrum. After fitting the resulting nine spectra to the B59 curve [Eq. (9)], he obtained a value of $q_{B}=3.9 \pm 1.5$. A similar experimental determination was done by Oakey (1982), who selected the 16 turbulent patches where the signal-to-noise ratio (SNR) for the shear velocity was the largest. After the scalar spectra for individual segments were calculated, they were assembled together in a nondimensional form and fitted to the theoretical shape of B59 within the range of the nondimensional wavenumber $\hat{k}_{d \theta}$ between 0.06 and 0.9 and found to be $q_{B}=3.7$. The same author found that when the scalar spectra are fitted one by one to the B59 model, the mean and standard deviation of the $16 q$ values is $q_{B}=3.67 \pm 1.52$. Nash and Moum (2002) took simultaneous measurements of shear, temperature, and conductivity in 400 patches to obtain thermal and salinity spectra. They fitted spectra individually to the B59 and K68 models, with the arithmetic means being $q_{B}=6.41$ and $q_{K}=8.54$ and the geometric means being $q_{B}=5.65$ and $q_{K}=7.5$, and used the geometric means to calculate the turbulent fluxes. The individual estimates of $q$ are within a dispersion range of one order of magnitude larger than the mean value.

All the experimental determinations of $q$ are with oceanographic data, and as far as we know no limnological or laboratory measurements are available. In the case of stationary turbulence with low wavenumber forcing, Bogucki et al. (1997) gave numerical results for a passive scalar advected by turbulence of $q_{B}=3.9 \pm 0.25$ and $q_{K}=5.26 \pm 0.25$. For numerical results of decaying turbulence, Antonia and Orlandi (2003) found $q_{B}=2.89$ and $q_{K}=3.41$. In both works, the numerical results show better agreement with the K68 model than with the B59 model.

Different authors have also checked the lowest wavenumber where the B59 and K68 models can be applied: 


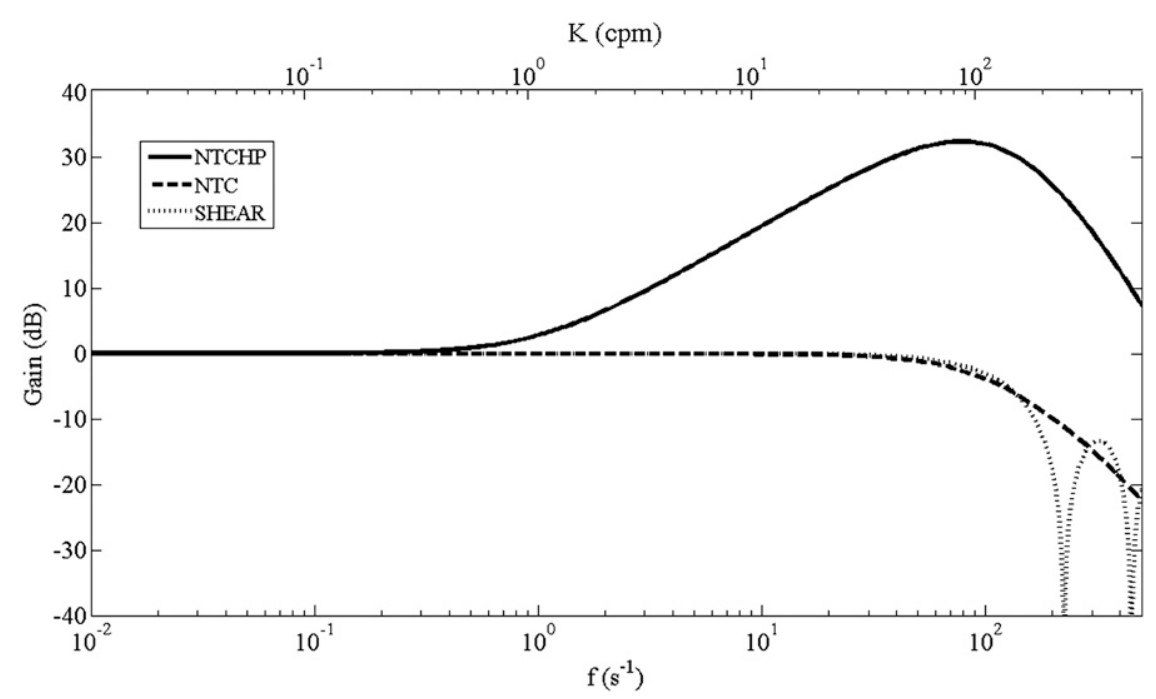

FIG. 2. Frequency response of the NTC and NTCHP output channels of the fast-response microthermistor as a function of the frequency $f$ (lower horizontal axis). And spatial response of the shear output channel as a function of the wavenumber $K$ (upper horizontal axis). The relationship between the frequency and the wavenumber is $K=f / W_{p}$ where $W_{p}=0.9 \mathrm{~m} \mathrm{~s}^{-1}$ is the profiling velocity.

that is, the wavenumber $k_{*}$ where the inertial-convective subrange in which $F_{\partial \theta}=C_{1 \theta} \varepsilon_{\theta} \varepsilon^{-1 / 3} k_{3}^{1 / 3}$, where $C_{1 \theta} \approx 0.4$ is the 1D Obukhov-Corrsin constant (Sreenivasan 1996), shifts to the viscous-convective subrange, where $F_{\partial \theta}=$ $q \varepsilon_{\theta} \varepsilon^{-1 / 2} \nu^{1 / 2} k_{3}$ [obtained from Eqs. (8)-(10) at large wavenumbers]. Grant et al. (1968) measured a mean value of $k_{*} / k_{d}=2.4 \times 10^{-2}$ or $k_{*} / k_{d \theta}=7.9 \times 10^{-3}(\mathrm{Pr}=7)$, with $k_{d}$ and $k_{\mathrm{d} \theta}$ being the Kolmogorov and the Batchelor wavenumbers. Dillon and Caldwell (1980) argued that there is no reason to expect the inertial subrange parameters to depend on the Prandtl number and determined, based on existing results in air, that $k_{*} / k_{d}=5.5 \times 10^{-2}$ or $k_{*} / k_{d \theta}=2.1 \times 10^{-2}$. Gibson et al. (1970), based on laboratory measurements, found $k_{*} / k_{d}$ between $3 \times 10^{-2}$ and $4 \times 10^{-2}$, which corresponds to $k_{*} / k_{d \theta}$ between $1 \times 10^{-2}$ and $2 \times 10^{-2}$. Steinbuck et al. (2009) proposed considering the lower wavenumber, $k_{*}=4 \times 10^{-2} k_{d}$ or $k_{*}=2 \times$ $10^{-2} k_{d \theta}$, for the experimental scalar spectrum to be fitted to the B59 model.

\section{Methods}

\section{a. Measurements and instrumentation}

Measurements were recorded with a free-falling MST profiler (Prandke and Stips 1998) sinking at an approximate velocity of $0.9 \mathrm{~m} \mathrm{~s}^{-1}$, which is within the optimal operational range for the shear probe, and sampling at $1024 \mathrm{~s}^{-1}$. This is a standard conductivity-temperaturedepth (CTD) profiler with microstructure temperature and small-scale shear sensors (fast-response sensors) as well. The shear measurements are made with an airfoil, PNS98 (manufactured by ISW Wassermesstechnik), which measures the lift force at its nose, and has a piezoceramic bending beam for a sensing element, with an electrical output calibrated to give one of the velocity components transverse to the profiling direction. The spatial resolution for the shear probe is defined based on the wavenumber where the response of the shear sensor drops $3 \mathrm{~dB}$. From Fig. 2, it is observed that this happens at around 100 cycles per meter (cpm) so its spatial resolution is about $1 \mathrm{~cm}$. The instrument used to measure fast-response temperature is basically a FP07 glass rod microthermistor with a sensitivity of $0.001^{\circ} \mathrm{C}$. Its nominal time constant is $7 \mathrm{~ms}$, although it has been documented that it is actually longer (Prandke 2005). A pre-emphasized analog channel has been added at the microthermistor by the manufacturer to achieve better signal-to-noise ratio at higher frequencies. After pre-emphasis and before calculating the spectra, data have been deemphasized in the Fourier space. Figure 2 shows the frequency response for the standard channel Negative Temperature Coefficient (NTC), the pre-emphasized channel NTC High Pass (NTCHP), and the spatial response of the shear channel.

For this study, we used 373 profiles measured in the western part of the northern lobe of Lake Banyoles in Catalonia, Spain (the general dynamic of the lake has been reviewed by Casamitjana et al. 2006). The lake has a surface area of $1.2 \mathrm{~km}^{2}$, a maximum depth of $46.4 \mathrm{~m}$, and a mean depth of $14.8 \mathrm{~m}$. At the measuring station, the total depth was $12 \mathrm{~m}$. The campaign was carried out 
a)

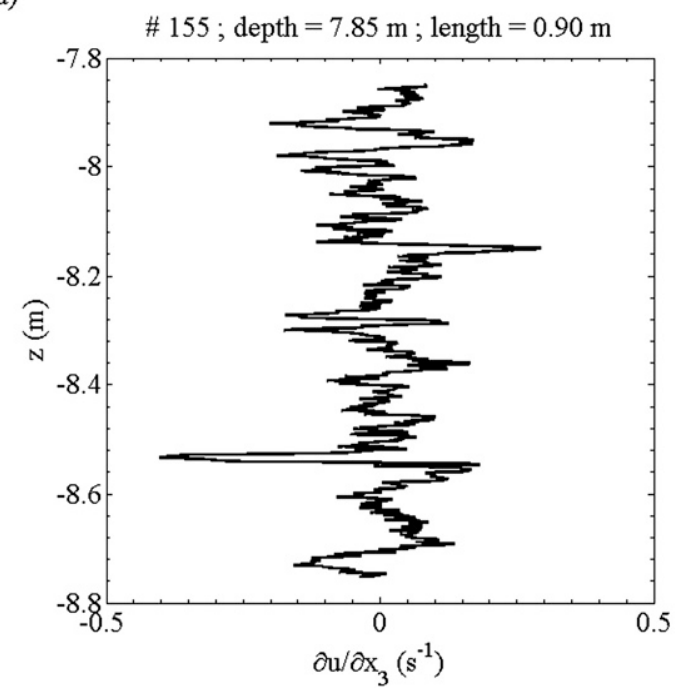

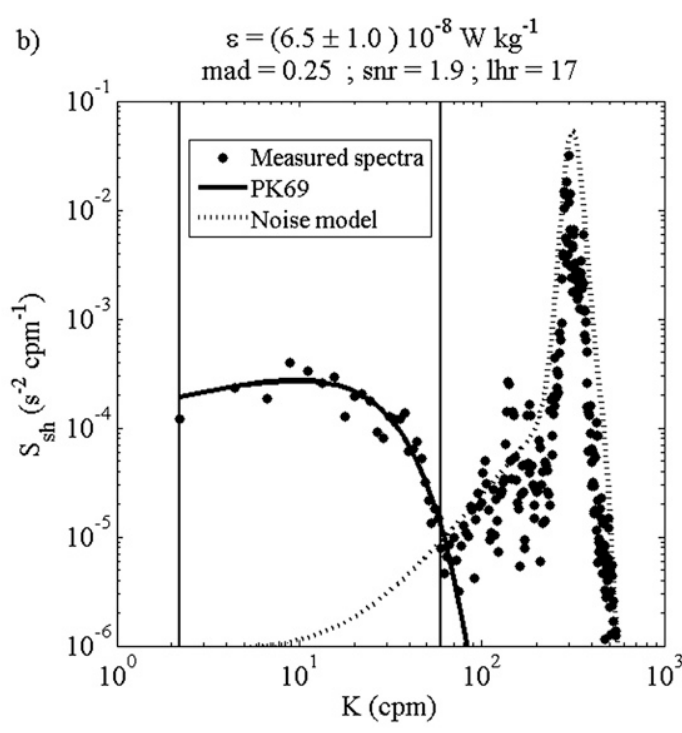

FIG. 3. (a) Measured shear profile in one of the homogeneous segments. (b) Resultant shear spectra as a function of the cpm wavenumber (points), with the best fit of the PK69 model (solid line) and the noise model (dotted line). Vertical bars correspond to the fitting interval.

from 27 June until 1 July 2009. During the campaign, the 30-min mean wind velocities were always below $3 \mathrm{~m} \mathrm{~s}^{-1}$. In summer, the lake is stratified, the internal seiches are permanently active, and the first and second vertical modes are excited (Roget et al. 1997), as was the case during the campaign. The same data are used to study the mesoscale intermittency within the main sheared thermocline of the lake (Planella et al. 2011).

\section{b. Data segmentation}

For this study, we used vertical segments of data of equal length where turbulence can be considered to be approximately homogeneous. All segments contained 1024 data points, which is equivalent to a length of about $0.9 \mathrm{~m}$. To check homogeneity, every segment was divided into seven subsegments of 256 points (so they overlapped at 128 points), and the variances of the temperature gradient and the small-scale shear were calculated for each one. If the variances at all subsegments remained in the same order of magnitude (ratio between variances at all subsegments smaller than 10), the segment was preliminarily accepted for this study. The number of preliminarily accepted homogeneous segments was $\approx 1000$.

Figure 3a shows an example of the measured shear velocity signal $\partial u / \partial x_{3}$ for an accepted segment, and in Fig. $3 b$ it is represented by the corresponding spectrum $S_{\mathrm{sh}}^{\exp }(K)$ (with solid circles) as a function of the onedimensional wavenumber $K$ in cpm: that is, $K=k_{3} / 2 \pi$. The spectrum was computed with Welch's periodogram method, considering three windowed subsegments of 512 data points $(0.45 \mathrm{~m})$. The solid line in Fig. $3 \mathrm{~b}$ is the best fit of the PK69 shear spectrum model to the experimental data. The noise model, which has been obtained from the quiet segments recorded during the campaign, is drawn as a dotted line. In Fig. 4a, small-scale temperature gradient data $\partial \theta / \partial x_{3}$ for the same segment considered in Fig. 3 is shown. In Fig. 4b, the experimental spectrum computed from these data $S_{\partial \theta}^{\exp }(K)$ is represented together with the curves of the theoretical B59 (solid line) and K68 (dashed line) models that we want to compare with measurements in this study. The noise model is drawn as a dotted line.

\section{c. Obtaining $\varepsilon$ from the best fit between experimental shear spectra and the PK69 model}

The PK69 model predicts the 3D energy spectra for the universal equilibrium range. From this model, the corresponding shear spectrum, which also depends on $\varepsilon$, can be calculated by integration (Hinze 1975). Unlike the case of the one-dimensional scalar spectrum, the shear spectrum does not have an analytical solution, but Roget et al. (2006) fitted an analytical function to the nondimensional numerically integrated result. Accordingly, the shear spectrum $F_{\mathrm{sh}}^{\mathrm{PK} 69}\left(k_{3}\right)$ in the PK69 model can be written as $F_{\text {sh }}^{\mathrm{PK} 69}\left(k_{3}\right)=\nu^{2} k_{d}^{3} \tilde{F}_{\text {sh }}^{\mathrm{PK} 69}\left(\tilde{k}_{3}\right)$, where $\tilde{k}_{3}=k_{3} / k_{d}$ is the nondimensional wavenumber and $\tilde{F}_{\text {sh }}^{\text {PK69 }}\left(\tilde{k}_{3}\right)$ is the nondimensional spectrum with the form

$\tilde{F}_{\text {sh }}^{\text {PK69 }}\left(\tilde{k}_{3}\right)=0.9372 \tilde{k}_{3}^{0.3748} \exp \left(-6.011 \tilde{k}_{3}^{1.548}\right)$. 
a)

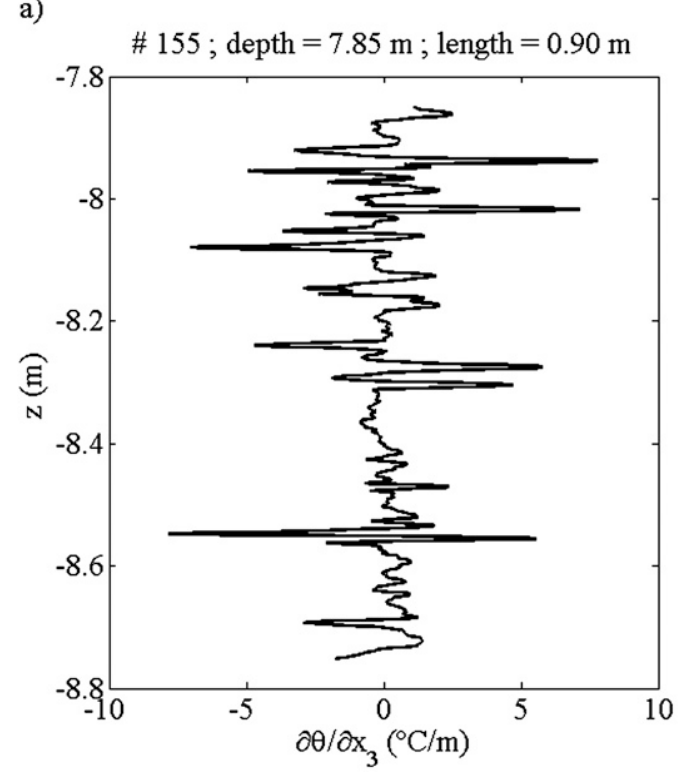

b)

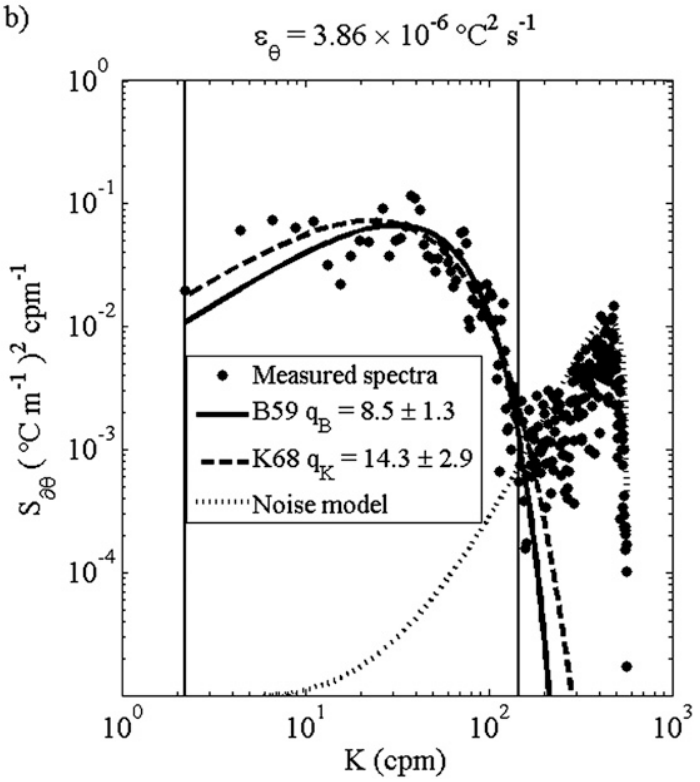

FIG. 4. (a) Measured temperature gradient profile of the same segment as in Fig. 3. (b) Corresponding temperature gradient spectra as a function of the cpm wavenumber (points), with the best fit of the B59 (solid line), K68 (dashed line), and noise (dotted line) models. The fit statistics for B59 are MAD $=0.47$ and LHR $=67$, and the fit statistics for $\mathrm{K} 68$ are $\mathrm{MAD}=0.44, \mathrm{LHR}=68$, and $\mathrm{SNR}=3$ (common for both models). Vertical bars correspond to the fitting interval.

The relationships between the angular magnitudes of the wavenumbers, $k_{3}$ and $F_{\mathrm{sh}}^{\mathrm{PK} 69}\left(k_{3}\right)$, and the corresponding cpm wavenumber magnitudes that we used in this paper, $K$ and $S_{\mathrm{sh}}^{\mathrm{PK} 69}(K)$, are $K=k_{3} / 2 \pi$ and $S_{\mathrm{sh}}^{\mathrm{PK} 69}(K)=2 \pi \mathrm{F}_{\mathrm{sh}}^{\mathrm{PK} 69}$. This spectra has its peak in $K=0.125 k_{d} / 2 \pi$. Integration of the shear spectrum to the Kolmogorov wavenumber $K_{d}=k_{d} / 2 \pi$ (in cpm) gives $99.8 \%$ of the variance, whereas integration to $K=0.513 K_{d}$ gives $90 \%$. Accordingly, to resolve all the variance of the shear, the Kolmogorov wavenumber has to be less than or equal to the maximum spatial resolution of the sensor, which in our case corresponds to $K=100 \mathrm{cpm}$ (see section 3a).

To obtain direct estimate of $\varepsilon$ from the small-scale shear, we fitted the measured shear spectrum $S_{\mathrm{sh}}^{\exp }(K)$ to the model $S_{\mathrm{sh}}^{\mathrm{PK} 69}(K)$. The lower wavenumber $K_{L}$ of the range considered for the fit was the smallest we could solve due to the windowed subsegment size, which is about $2 \mathrm{cpm}$. The higher wavenumber considered for the fit $K_{U}$ varies for each segment, because it is the $K$ value where the experimental spectra $S_{\mathrm{sh}}^{\exp }(K)$ intersects the noise model (dotted line in Fig. $3 \mathrm{~b}$ where the borders of the fitted domain are indicated by two vertical bars). The best fit between experimental data and the PK69 model was obtained for each segment following the method of likelihood function optimization given by Ruddick et al. (2000) for the case of the temperature gradient, which has been adapted here for the case of shear. The likelihood function has a Gaussian shape, and the value of $\varepsilon$ that maximizes the likelihood function gives the best fit to the model. The error of the fitting parameter $\varepsilon$ with a confidence interval of $95 \%$ is given by the standard deviation multiplied by 1.96 . Accordingly, in the case of Fig. 3b the resulting value of $\varepsilon$ that gives the best fit is $\varepsilon=(6.5 \pm 1.0) \times 10^{-8} \mathrm{~W} \mathrm{Kg}^{-1}$ and the corresponding Kolmogorov and Batchelor wavenumbers are $K_{d}=$ $k_{d} / 2 \pi=70 \mathrm{cpm}$ and $K_{d \theta}=k_{d \theta} / 2 \pi=200 \mathrm{cpm}$.

Following Ruddick et al. (2000), we used three parameters to quantify the goodness of this fit: the mean absolute deviation (MAD) of the ratio between the experimental and theoretical spectra within the fitting dominium; the SNR; and the likelihood ratio (LHR), which describes if the measured spectrum fits the PK69 model or a power-law spectrum better. So, a good fit has a small MAD and large SNR and LHR. To accept a fit, Ruddick et al. (2000) suggested MAD $\leq 1.1, \mathrm{SNR} \geq 1.3$, and $\mathrm{LHR} \geq 2$. In this study, we require MAD $\leq 0.3$, a smaller value than that suggested by Ruddick, for a more precise fit. In the example given in Fig. 3b, the value of the three parameters are MAD $=0.25, \mathrm{SNR}=1.9$, and $\mathrm{LHR}=17$.

After fitting the experimental data to the theoretical PK69 model for individual segments and obtaining $\varepsilon$ for each one, nondimensional spectra can be obtained according to $\tilde{F}_{\mathrm{sh}}^{\exp }\left(\tilde{k}_{3}\right)=S_{\mathrm{sh}}^{\exp }\left(2 \pi v^{2} k_{d}^{3}\right)^{-1}$, where $\tilde{k}_{3}=k_{3} / k_{d}$, and they can be assembled together to check the validity of the model. The nondimensional shear spectrum will be shown in section 4 (results and discussion). 


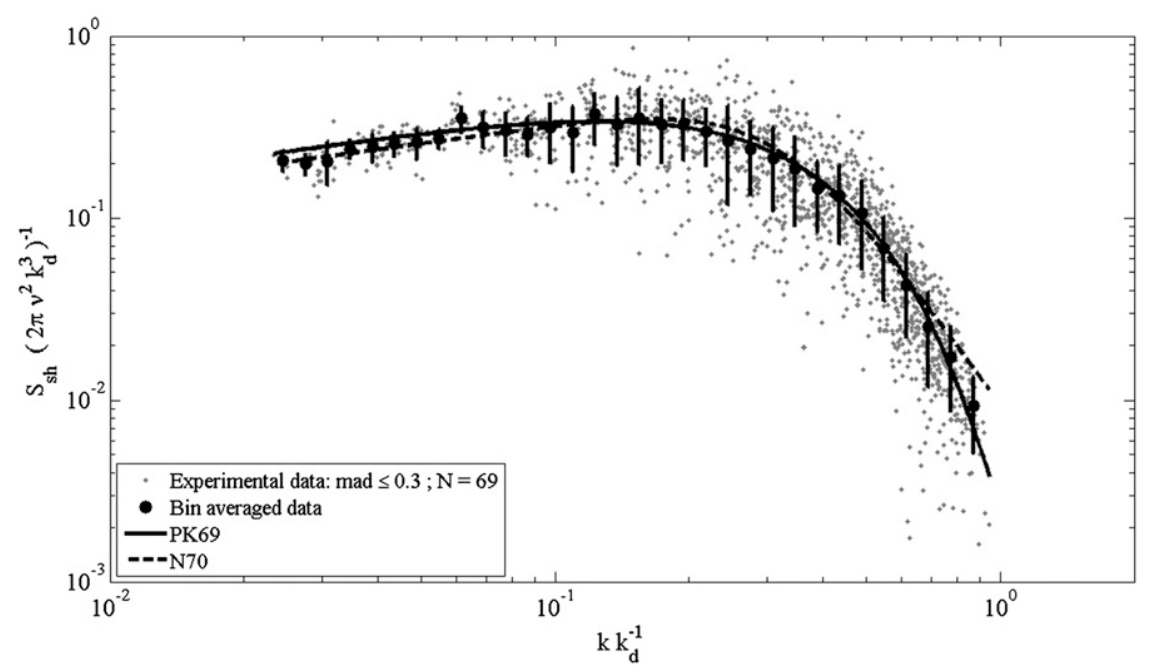

FIG. 5. Nondimensional shear spectra for the ensemble of 69 segments discussed in section 3c (points) as a function of the Kolmogorov wavenumber $\tilde{k}=k \times k_{d}^{-1}$ and the equivalent binaveraged data (solid circles) with their standard deviation bars. The PK69 theoretical and N70 experimental models are represented by solid and dashed lines.

\section{d. Obtaining $\varepsilon_{\theta}$ from the measured temperature gradient spectra and determining the turbulent parameter from individually fitted spectra}

After computation of the spectra of the gradient of the microstructure temperature $S_{\partial \theta}^{\exp }(K)$ for the 69 homogeneous turbulent segments where small-scale shear spectra could be accurately fitted to the PK69 model, the rate of dissipation of temperature variance $\varepsilon_{\theta}$ is obtained by integrating,

$$
\varepsilon_{\theta}=6 \kappa_{\theta} \int_{K_{L}}^{K_{U}} S_{\partial \theta}^{\exp }(K) d K,
$$

where, following Steinbuck et al. (2009), $2 \pi K_{L}$ (in $\mathrm{rad} \mathrm{\textrm {m } ^ { - 1 }}$ ) is equal to $k_{*}=2 \times 10^{-2} k_{d \theta}$. The wavenumber $k_{*}$ has been defined in section $2 \mathrm{~b}$ as the transition wavenumber from the inertial-convective to the viscousconvective subranges, and $k_{d \theta}$ is calculated from the $\varepsilon$ value obtained following the procedure described in section 3c. In the example shown in Fig. 4b, Eq. (12) gives $\varepsilon_{\theta}=3.82 \times 10^{-6}{ }^{\circ} \mathrm{C}^{2} \mathrm{~s}^{-1}$. Once $\varepsilon_{\theta}$ is calculated, $S_{\partial \theta}^{\exp }(K)$ can be fitted to both theoretical models, the $\mathrm{B} 59$ or the $\mathrm{K} 68$, for the one-dimensional temperature spectra following the same procedure described in section $3 \mathrm{c}$ for shear spectra. Note that, in cpm units, the theoretical spectra are $S_{\partial \theta}^{\mathrm{B} 59}(K)=2 \pi F_{\partial \theta}^{\mathrm{B} 59}$ and $S_{\partial \theta}^{\mathrm{K} 68}(K)=2 \pi F_{\partial \theta}^{\mathrm{K} 68}$, where $F_{\partial \theta}^{\mathrm{B} 59}\left(k_{3}\right)$ and $F_{\partial \theta}^{\mathrm{K} 68}\left(k_{3}\right)$ are given by Eqs. (8)-(10), and $K=k_{3} / 2 \pi$. In this case the only fitting parameter is $\phi=k_{d \theta} / \sqrt{2 q}$ and because $\varepsilon$ (or $k_{d \theta}$ ) is already known for every segment (section 3c), the value of $\phi$ providing the better fit also provides the turbulent parameter $q$.
Figure $4 \mathrm{~b}$ shows an example of the best fit of the measured temperature gradient spectra to models B59 (solid line) and K68 (dashed line), where the resulting turbulent parameters are $q_{B}=8.5 \pm 1.3$ and $q_{K}=14.3 \pm 2.9$. Statistics of the turbulent parameters obtained for each model are presented in section 4.

Once the thermal spectrum $S_{\partial \theta}^{\exp }(K)$ and $\varepsilon$ and $\varepsilon_{\theta}$ are known for each segment, individual spectrum can be nondimensionalized, according to $\hat{F}_{\partial \theta}^{\exp }(\hat{k})=S_{\partial \theta}^{\exp } 2 \kappa_{\theta} k_{d \theta}$ $\left(2 \pi \varepsilon_{\theta}\right)^{-1}$, where $\hat{k}=k / k_{d \theta}$ is the Batchelor nondimensional wavenumber, and plotted together as was done in Fig. 5 for the case of small-scale shear. Fitting the binaveraged nondimensional spectrum to each of the theoretical models (B59 and K68) will also provide an estimate of the turbulent parameter. Results are presented in the following section.

\section{Results and discussion}

Following procedures described in section $3 \mathrm{c}$ and using our data, we found 69 segments where the spectrum fits the PK69 model extremely well out of the 1000 homogeneous segments that were first determined (section $3 b)$. The turbulent kinetic energy dissipation rates of these segments range between $10^{-8}$ and $10^{-6.5} \mathrm{~W} \mathrm{~kg}^{-1}$. Note that for all the cases the Kolmogorov wavenumbers $K_{d}=k_{d} / 2 \pi$ range from 44 to $104 \mathrm{cpm}$, being less than or approximately equal to the maximum resolved wavenumber with the PNS98 airfoil of $100 \mathrm{cpm}$, as discussed in section 3c. This fact may indicate that the goodness of fit criteria in the shear data have automatically limited the range of dissipations, with $K_{d}=k_{d} / 2 \pi \leq 100 \mathrm{cpm}$. 

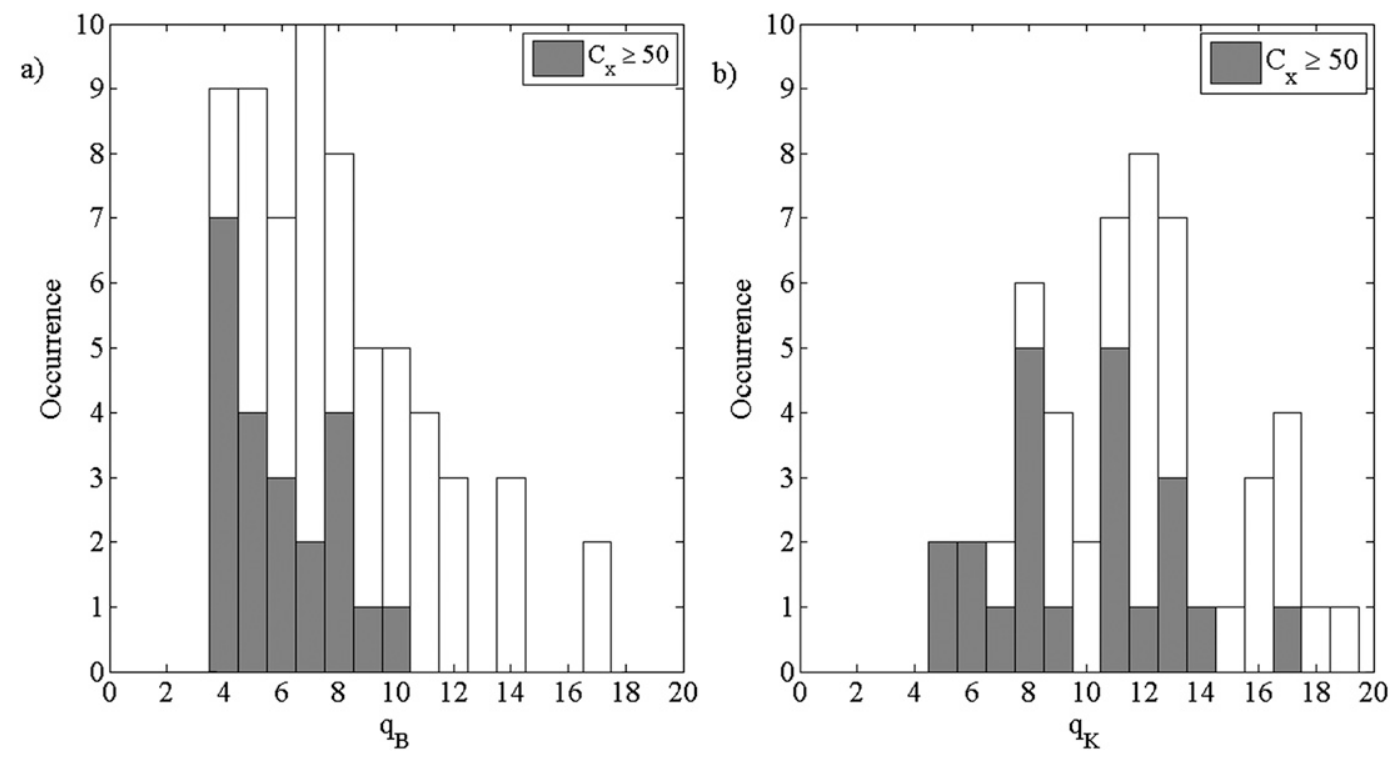

FIG. 6. (a) Occurrence of $q_{B}$ for individual fitting of thermal gradient spectra for the whole ensemble of 69 segments (solid line bars) and for $C_{x} \geq 50$ (gray bars) between 0 and 20. (b) As in Fig. 6a, but for $q_{K}$.

Figure 5 shows the nondimensional spectra assembled for the 69 segments used in this study. As observed, binaveraged spectra (solid circles) follow the PK69 model $\tilde{F}_{\text {sh }}^{\text {PK69 }}\left(\tilde{k}_{3}\right)$ given in Eq. (11) (solid line) and the N70 model (dashed line) well.

For the chosen segments, the $q$ values for the Batchelor and Kraichnan models have been obtained by fitting the temperature gradient spectra data to the theoretical curves as described in section $3 \mathrm{~d}$. The turbulent parameter $q_{B}$ obtained for the B59 model ranges between 3.5 and 57, and for the K68 model $q_{K}$ ranges between 5 and 130. However, to assure certain degree of isotropy (see section 1), these intervals are considerably reduced when only those segments with a Cox number larger than 50 are considered. This is the case of the 22 segments out of 69 for which $q_{B}$ ranges between 3 and 10 and $q_{K}$ ranges between 5 and 17. The occurrences of different values of the turbulent parameters obtained in the fitting process are represented in Fig. $6 \mathrm{a}\left(q_{B}\right)$ and Fig. $6 \mathrm{~b}\left(q_{K}\right)$ for the range of $q$ between 0 and 20, a range that includes all the values from $C_{x} \geq 50$ segments (gray bars in Fig. 6). There were four occurrences of $q$ values outside the represented range for segments with $C_{x}<50$ for $q_{B}(6 \%$ of the total) and 18 for $q_{K}$ (27\% of the total). For those segments where $C_{x} \geq 50$, the arithmetic mean of the turbulent parameters are $q_{B}=6.0 \pm 3.5$ and $q_{K}=9.8 \pm 6.4$. These values are consistent with those given by Nash and Moum (2002), who, following a similar procedure of individually fitting spectra, obtained mean values for the turbulent parameter of $q_{B}=6.41$ and $q_{K}=8.54$, but in their case the individual values were more scattered around the mean (about 5 times the mean value). The dispersion in $q$ decreases when the Cox numbers increase (not shown), which can be expected considering that the turbulent parameter $q$ is defined only for isotropic turbulence. In fact, the B59 and K68 models have been developed for isotropic turbulence too.

Alternatively, according to section $3 \mathrm{~d}$, the nondimensional spectra can be computed knowing $\varepsilon$ and $\varepsilon_{\theta}$. In Fig. 7a, the whole ensemble of the 69 nondimensional spectra has been represented (points) together with the corresponding bin-averaged spectra (solid circles). From Eq. (8), the nondimensional spectra should follow $\sqrt{2 q} f_{\partial \theta}\left(y_{3}\right)$, where the function $f_{\partial \theta}(y 3)$ is given by Eqs. (9) and (10) for the B59 and K68 models. Accordingly, a value of $q$ can be obtained from the best fit between the band-averaged nondimensional measured spectra and the theoretical curves $\sqrt{2 q} f_{\partial \theta}\left(y_{3}\right)$. In Fig. 7a, the best fits, which were computed within the range $\hat{k}=0.025$ and $\hat{k} \approx 1$, following the procedure of Ruddick et al. (2000), are represented by a solid line in the case of the Batchelor model and by a dashed line for the Kraichnan model. The values of the fit parameters, $q_{B}$ and $q_{K}$, that give the best likelihood were $q_{B}=5.0 \pm 0.7$ and $q_{K}=9.9 \pm 2.4$, where the error has been estimated from the Gaussian-shaped likelihood function.

The same procedure has been followed to produce the results given in Fig. $7 \mathrm{~b}$ with the 22 spectra that meet the requirement $C_{x} \geq 50$ and, in this case, the turbulent parameters that gave the best fit were found to be $q_{B}=$ $4.4 \pm 0.8$ and $q_{K}=7.9 \pm 2.5$. Errors of $q$ obtained from individually fitting spectrum results are larger than those 



FIG. 7. (a) Nondimensional spectra of the temperature gradient as a function of the Batchelor nondimensional wavenumber $\hat{k}=k \times k_{d \theta}^{-1}$ for the whole ensemble of 69 segments (points); the bin-averaged spectra (solid circles) with the corresponding standard deviation (error bars); and the best fit to the theoretical models of B59 (solid) and K68 (dashed), giving the corresponding turbulent parameters $q_{B}$ and $q_{K}$, shown in the legend. Also, the Oakey (1982) oceanic data (open squares) have been incorporated. (b) As in Fig. 6a, but for the 22 segments with $C_{x} \geq 50$.

obtained from the nondimensional spectra, but results obtained with both procedures, when their error interval is taken into consideration, are compatible. Furthermore, these values are in agreement with those obtained by Grant et al. (1968) and Oakey (1982), with $q_{B} \approx 4$, which were discussed in section $2 \mathrm{~b}$. The nondimensional spectra measured by Oakey (1982) has also been represented in Fig. 7 (open squares), showing good agreement with the present data (solid circles), especially in the case of Fig. $7 \mathrm{~b}$, where the value of Cox numbers was also $C_{x} \geq 50$.
Also from Fig. 7, it is observed that experimental data follow the K68 model better than the B59 model, although in Fig. 7a (all Cox) the K68 model follows the experimental data only for $\hat{k}>0.1$. In Fig. $7 \mathrm{~b}\left(C_{x} \geq 50\right)$, the K68 model fits our experimental data for $\hat{k}>0.04$. That is, Figs. 7a,b show that the validity of the B59 and K68 models is related to the degree of isotropy of the turbulence, which can be evaluated either by the Cox or the buoyancy Reynolds numbers, as discussed in section 1. 
TABLE 1. Different statistical magnitudes relating $\varepsilon^{\text {th }}$ and $\varepsilon^{\text {sh }}$.

\begin{tabular}{lccccc}
\hline & $q$ & $\frac{\overline{\varepsilon^{\text {th }}}}{\varepsilon^{\text {sh }}}$ & $\min \left(\frac{\varepsilon^{\text {th }}}{\varepsilon^{\text {sh }}}\right)$ & $\max \left(\frac{\varepsilon^{\text {th }}}{\varepsilon^{\text {sh }}}\right)$ & $\operatorname{std}\left(\frac{\varepsilon^{\text {th }}}{\varepsilon^{\text {sh }}}\right)$ \\
\hline B59 & 4.4 & 0.70 & 0.20 & 1.30 & 0.36 \\
B59 & 3.7 & 0.49 & 0.14 & 0.92 & 0.26 \\
K68 & 7.9 & 0.90 & 0.21 & 2.43 & 0.63 \\
K68 & 7.5 & 0.82 & 0.19 & 2.19 & 0.57 \\
\hline
\end{tabular}

To check the statistical relevance of the models and the value of the turbulent parameter to determine $\varepsilon$, we have fitted the thermal gradient spectra computed from measurements to the B59 and K68 models given in Eqs. (8)-(10), and we have found the rate of dissipation of turbulent kinetic energy $\varepsilon$ from the microstructure thermal signal, which we will denote as $\varepsilon^{\text {th }}$, and $\varepsilon^{\text {th }}$ is compared with the value of $\varepsilon$ obtained from the shear spectra $\varepsilon^{\text {sh }}$ (section $3 \mathrm{c}$ ). This has been done for the most isotropic datasets with $C_{x} \geq 50$ using the values $q_{B}=4.4$ (present data value) and $q_{B}=3.7$ (Oakey 1982) for B59 and $q_{K}=7.9$ (present data value) and $q_{K}=7.5$ (Nash and Moum 2002) for K68. Different statistical magnitudes relating the estimates of $\varepsilon$ in the two different approaches are shown in Table 1. The six columns of the table stand for the model (B59 or K68); the value of the turbulent parameter $q$ used in the fitting procedure; and the mean $\left(^{-}\right)$, minimum ( $\left.\min \right)$, maximum ( $\max$ ), and standard deviation (std) of the ratio $\varepsilon^{\text {th }} / \varepsilon^{\text {sh }}$. As observed, the ratio $\overline{\varepsilon^{\text {th }} / \varepsilon^{\text {sh }}}$ is closer to 1 in the case of the Kraichnan model with $q_{K}=7.9$ (value from section 4 of Fig. 7b) than for K68 with $q_{K}=7.5$ (from Nash and Moum 2002) and B59 with $q_{B}=4.4$ (from this work) or $q_{B}=3.7$ (from Oakey 1982 ), which underestimates $\varepsilon^{\text {th }}$ by $18 \%, 51 \%$, and $30 \%$ of the true value $\left(\varepsilon^{\mathrm{sh}}\right)$. In the best estimate case, K68 with $q_{K}=7.9$, the individual values of $\varepsilon^{\text {th }}$ have their maximum underestimation and overestimation in a factor of $5(\mathrm{~min}=0.21$ in Table 1$)$ and 2.43 , respectively (max in Table 1). Kocsis et al. (1999) did a similar analysis, with B59 and $q_{B}=3.4$, finding $\overline{\varepsilon^{\text {th }}} / \overline{\varepsilon^{\text {sh }}}=1.2$, and maximum deviation factors of 10 , but they covered a wider range of $\varepsilon^{\text {sh }}$ (from $10^{-9}$ to $10^{-6}$ ) than the present work (from $10^{-8}$ to $10^{-6.5}$ ).

Regarding isotropy, in Fig. 8 the Cox number $C_{x}$ of the 69 studied segments is represented (open circles) as a function of the corresponding buoyancy Reynolds number $\mathrm{Re}_{b}$ in logarithmic scales. Segments with $C_{x} \geq 10$ are highlighted with solid circles. For this last case, a linear fit was obtained-with a correlation of $r^{2}=0.71$-and represented with a solid line. The resulting line gives $C_{x}=1.36 \times \mathrm{Re}_{b}^{0.96}$, which is basically equivalent to $C_{x} \propto$ $\mathrm{Re}_{b}$, as argued by Dillon and Caldwell (1980) (commented in section 1). In fact, this linear relationship between the Cox and the Reynolds numbers is equivalent to a constant mixing efficiency $\Gamma$, because according to Osborn and Cox (1972) $\Gamma=\kappa_{\theta} C_{x} N^{2} / \varepsilon$, which is the same as $\Gamma=\operatorname{Pr}^{-1} C_{x} / \operatorname{Re}_{b}$. In the case of our data, $C_{x} / \operatorname{Re}_{b} \approx 1.36$ and $\Gamma \approx 1.36 / 7=0.19$. Alternatively, if it is calculated for each segment it is found that the mean and error gives $\Gamma=0.21 \pm 0.17$. These results are in agreement with the value of 0.2 normally used for active turbulence (Oakey 1982; Roget et al. 2006) and even the lower value of 0.16 given by Ravens et al. (2000) for limnetic waters.

\section{Overview and conclusions}

The spectral models of B59 and K68 were developed for a scalar field, like temperature or any concentration, which is advected by an isotropic velocity field, but whose buoyancy forces by themselves do not affect the

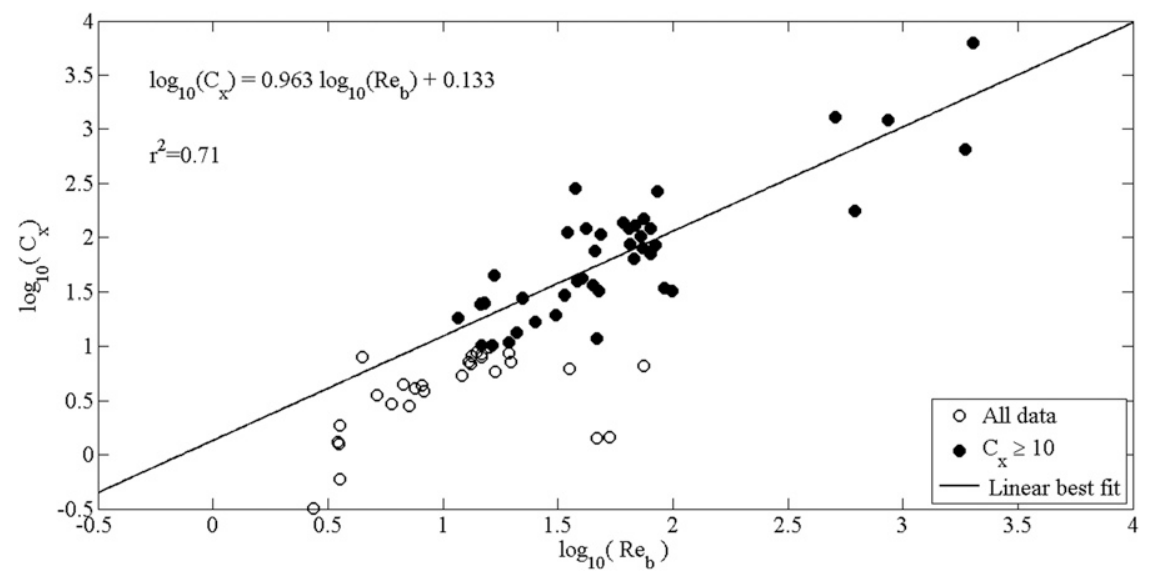

FIG. 8. Cox number as a function of the buoyancy Reynolds number (logarithmic scales) for the whole ensemble of 69 accepted segments. Solid circles stand for $C_{x} \geq 10$, which follows a linear dependence (solid line). 
velocity field. Therefore, it has passive behavior and should show isotropy. Below the two spectrum models, there are robust and different physical basins. The Batchelor model assumes that a locally uniform strain rate with slow time variability advects the scalar, and the Kraichnan model assumes a small-scale velocity field with an infinitely short correlation time so very fast time variability. Both of them depend on a turbulent parameter, which has to be determined experimentally. Above a certain degree of isotropy, quantified by the Cox and the buoyancy Reynolds numbers, and within the error, the experimental data agree with both models, but they follow the Kraichnan model better than the Batchelor model. In particular, for the larger values of our measured Cox numbers, $C_{x} \geq 50$, our data accurately fit the Kraichnan nondimensional spectra, for almost two orders of magnitude of the nondimensional wavenumber, including part of the viscous-convective subrange, but it does not fit the Batchelor model so accurately. From the fit between our experimental data and the spectrum models and for $C_{x} \geq 50$, the turbulent parameters are $q_{K}=7.9 \pm$ 2.5 for the Kraichnan model and $q_{B}=4.4 \pm 0.8$ for the Batchelor model, which are both coherent with results from previous experimental studies in the ocean. When considering spectra with $C_{x}<50$, the Kraichnan spectra only fit the experimental data at the largest wavenumber values and the estimated turbulent parameter increases. This deviation from the Kraichnan model for small isotropy can be justified by the fact that the model assumes the isotropy condition. Present data show a proportionality between the Cox and Reynolds numbers, a fact that confirms a constant value of $\approx 0.2$ for the mixing efficiency expected for active turbulence. Determining $\varepsilon$ from the thermal spectrum fit shows better agreement with the $\varepsilon$ obtained directly from the shear spectra for the Kraichnan model, with $q_{K}=7.9$, than for the Batchelor model, with $q_{B}=3.7$ or $q_{B}=4.4$, which both underestimate the value of $\varepsilon$. Because dissipations in this study are from $10^{-8}$ to $10^{-6.5}$ and Cox numbers are relatively small, future studies of the K68 and B59 models and the turbulent parameter should consider a wider range of dissipations and Cox numbers. In any case, Nash and Moum (2002) and the present study both confirm that the K68 model is more accurate than the B59 model for dissipation estimates and consequently turbulent fluxes from microstructure temperature data.

Acknowledgments. This research was developed under Spanish Government Project FIS2008-03608. We would also like to thank Francesc Nogué (University of Girona) for his electronic engineering support and Joan Corominas (Banyoles) for his sailing support.

\section{REFERENCES}

Antonia, R. A., and P. Orlandi, 2003: On the Batchelor constant in decaying isotropic turbulence. Phys. Fluids, 15, 2084, doi:10.1063/ 1.1577346 .

Batchelor, G., 1959: Small-scale variation of convected quantities like temperature in turbulent fluid. 1. General discussion and the case of small conductivity. J. Fluid Mech., 5, 113-133.

Bogucki, D., J. A. Domaradzki, and P. K. Yeung, 1997: Direct numerical simulations of passive scalars with $\operatorname{Pr}>1$ advected by turbulent flow. J. Fluid Mech., 343, 111-130.

Casamitjana, X., J. Colomer, E. Roget, and T. Serra, 2006: Physical limnology in Lake Banyoles. Limnetica, 25, 181-188.

Corrsin, S., 1951: On the spectrum of isotropic temperature fluctuations in an isotropic turbulence. J. Appl. Phys., 22, 469-473.

Dillon, T. M., 1982: Vertical overturns-A comparison of Thorpe and Ozmidov length scales. J. Geophys. Res., 87, 9601-9613.

— , and D. R. Caldwell, 1980: The Batchelor spectrum and dissipation in the upper ocean. J. Geophys. Res., 85, 1910-1916.

Gargett, A. E., T. R. Osborn, and P. W. Nasmyth, 1984: Local isotropy and the decay of turbulence in a stratified fluid. J. Fluid Mech., 144, 231-280.

Gibson, C. H., 1968: Fine structure of scalar fields mixed by turbulence. 2. Spectral theory. Phys. Fluids, 11, 2316-2327.

— of turbulent velocity and scalar fields. J. Fluid Mech., 16, 365-384.

$\longrightarrow$, R. R. Lyon, and I. Hirschsohn, 1970: Reaction product fluctuations in a sphere wake. AIAA J., 8, 1859-1863.

Grant, H. L., B. A. Hughes, W. M. Vogel, and A. Moilliet, 1968: Spectrum of temperature fluctuations in turbulent flow. J. Fluid Mech., 34, 423-442.

Gregg, M. C., 1999: Uncertainties and limitations in measuring $\epsilon$ and $\chi_{T}$. J. Atmos. Oceanic Technol., 16, 1483-1490.

_ , and J. K. Horne, 2009: Turbulence, acoustic backscatter, and pelagic nekton in Monterey Bay. J. Phys. Oceanogr., 39, 10971114.

Hinze, J. O., 1975: Turbulence. 2nd ed. McGraw-Hill, 790 pp.

Jonas, T., A. Stips, W. Eugster, and A. Wüest, 2003: Observations of a quasi shear-free lacustrine convective boundary layer: Stratification and its implications on turbulence. J. Geophys. Res., 108, 3328, doi:10.1029/2002JC001440.

Kocsis, O., H. Prandke, A. Stips, A. Simon, and A. Wüest, 1999: Comparison of dissipation of turbulent kinetic energy determined from shear and temperature microstructure. J. Mar. Syst., 21, 67-84.

Kolmogorov, A. N., 1941: The local structure of turbulence in an incompressible viscous fluid for very large Reynolds number. C. R. Acad. Sci. USSR, 30, 301.

Kraichnan, R. H., 1968: Small-scale structure of a scalar field convected by turbulence. Phys. Fluids, 11, 945-953.

_ 1974: Convection of a passive scalar by a quasi-uniform random straining field. J. Fluid Mech., 64, 737-762.

Kundu, P. K., 2008: Fluid Mechanics. 4th ed. Academic Press, 872 pp.

Lozovatsky, I. D., T. M. Dillon, A. Y. Erofeev, and V. N. Nabatov, 1999: Variations of thermohaline structure and turbulent mixing on the Black Sea shelf at the beginning of autumn cooling. J. Mar. Syst., 21, 255-282.

Luketina, D. A., and R. Imberger, 2001: Determining turbulent kinetic energy dissipation from Batchelor curve fitting. J. Atmos. Oceanic Technol., 18, 100-113. 
Nash, J. D., and J. N. Moum, 2002: Microstructure estimates of turbulent salinity flux and the dissipation spectrum of salinity. J. Phys. Oceanogr., 32, 2312-2333.

Nasmyth, P. W., 1970: Oceanic turbulence. Ph.D. dissertation, British Columbia University, $69 \mathrm{pp}$.

Oakey, N. S., 1982: Determination of the rate of dissipation of turbulent energy from simultaneous temperature and velocity shear microstructure measurements. J. Phys. Oceanogr., 12, 256-271.

Obukhov, A. M., 1949: Structure of the temperature field in a turbulent flow. Izv. Akad. Nauk SSSR, Ser. Geogr. Geofiz., 13, $58-69$.

Osborn, T. R., 1980: Estimates of the local-rate of vertical diffusion from dissipation measurements. J. Phys. Oceanogr., 10, 83-89.

— Dyn., 3, 321-345.

Panchev, S., and D. Kesich, 1969: Energy spectrum of isotropic turbulence at large wave-numbers. C. R. Acad. Bulg. Sci., 22, 627.

Piera, J., E. Roget, and J. Catalan, 2002: Turbulent patch identification in microstructure profiles: A method based on wavelet denoising and Thorpe displacement analysis. J. Atmos. Oceanic Technol., 19, 1390-1402.

Planella, J., E. Roget, and I. Lozovatsky, 2011: Statistics of Microstructure Patchiness in a Stratified Lake. J. Geophys. Res., doi:10.1029/2010JC006911, in press.

Prandke, H., 2005: Microstructure sensors. Marine Turbulence: Theories, Observations and Models, H. Baumert, J. Simpson, and J. Sündermann, Eds., Cambridge University Press, 101109.

_ microstructure-turbulence profiler: Detection limit of dissipation rates. Aquat. Sci., 60, 191-209.

Ravens, T. M., O. Kocsis, A. Wüest, and N. Granin, 2000: Smallscale turbulence and vertical mixing in Lake Baikal. Limnol. Oceanogr., 45, 159-173.
Roget, E., G. Salvade, and F. Zamboni, 1997: Internal seiche climatology in a small lake where transversal and second vertical modes are usually observed. Limnol. Oceanogr., 42, 663-673.

_ I. Lozovatsky, X. Sanchez, and M. Figueroa, 2006: Microstructure measurements in natural waters: Methodology and applications. Prog. Oceanogr., 70, 126-148, doi:10.1016/j.pocean. 2006.07.003.

Ruddick, B., A. Anis, and K. Thompson, 2000: Maximum likelihood spectral fitting: The Batchelor spectrum. J. Atmos. Oceanic Technol., 17, 1541-1555.

Saggio, A., and J. Imberger, 2001: Mixing and turbulent fluxes in the metalimnion of a stratified lake. Limnol. Oceanogr., 46, 392-409.

Sanchez, X., and E. Roget, 2007: Microstructure measurements and heat flux calculations of a triple-diffusive process in a lake within the diffusive layer convection regime. J. Geophy. Res., 112, C02012, doi:10.1029/2006JC003750.

Smyth, W. D., J. N. Moum, and D. R. Caldwell, 2001: The efficiency of mixing in turbulent patches: Inferences from direct simulations and microstructure observations. J. Phys. Oceanogr., 31, 1969-1992.

Sreenivasan, K. R., 1996: The passive scalar spectrum and the Obukhov-Corrsin constant. Phys. Fluids, 8, 189-196.

Steinbuck, J. V., M. T. Stacey, and S. G. Monismith, 2009: An Evaluation of $\chi_{T}$ estimation techniques: Implications for batchelor fitting and $\varepsilon$. J. Atmos. Oceanic Technol., 26, 16521662.

Stevens, C., M. Smith, and A. Ross, 1999: SCAMP: Measuring turbulence in estuaries, lakes and coastal waters. Water Atmos., 7, 20-21.

Tennekes, H., and J. L. Lumley, 1972: A First Course in Turbulence. MIT Press, $300 \mathrm{pp}$.

Wüest, A., D. C. van Senden, J. Imberger, G. Piepke, and M. Gloor, 1996: Comparison of diapycnal diffusivity measured by tracer and microstructure techniques. Dyn. Atmos. Oceans, 24, 27-39. 